\title{
RAS genes in colorectal carcinoma: pathogenesis, testing guidelines and treatment implications
}

\author{
Omer Saeed, ${ }^{1}$ Antonio Lopez-Beltran, ${ }^{2}$ Kurt W Fisher, ${ }^{3}$ Marina Scarpelli, ${ }^{4}$ \\ Rodolfo Montironi, ${ }^{4}$ Alessia Cimadamore, ${ }^{4}$ Francesco Massari, ${ }^{5}$ Matteo Santoni, ${ }^{6}$ \\ Liang Cheng ${ }^{1}$
}

'Department of Pathology and Laboratory Medicine, Indiana University School of Medicine, Indianapolis, USA

${ }^{2}$ Department of Pathology and Surgery, Faculty of Medicine, Cordoba, Spain

${ }^{3}$ Department of Pathology and Microbiology, University of Nebraska Medical Center, Omaha, USA

${ }^{4}$ Section of Pathological Anatomy, Polytechnic University of the Marche Region, School of Medicine, United Hospitals, Ancona, Italy

${ }^{5}$ Division of Oncology, S. OrsolaMalpighi Hospital, Bologna, Italy ${ }^{6}$ Oncology Unit, Macerata Hospital, Macerata, Italy

\section{Correspondence to} Dr Liang Cheng, Department of Pathology and Laboratory Medicine, Indiana University School of Medicine,

Indianapolis, IN 46202, USA liang_cheng@yahoo.com

Received 28 August 2018 Revised 3 October 2018 Accepted 4 October 2018 Published Online First 13 November 2018

Check for updates

(C) Author(s) (or their employer(s)) 2019. No commercial re-use. See rights and permissions. Published by BMJ.

To cite: Saeed O, Lopez-

Beltran A, Fisher KW,

et al. J Clin Pathol

2019:72:135-139.

\section{ABSTRACT}

The RAS family is among the most commonly mutated genes in all human malignancies including colon cancer. In normal cells, RAS proteins act as a link in the intracellular signal transduction initiated by binding of growth factors to cell membrane receptors mediating cell survival. RAS isoproteins have great morphological similarities, but despite that, they are thought to have different functions in different tissues. RAS mutations, as supported by several studies including animal models, have a role in the development and progression of colorectal cancer. The detection of RAS mutations in patients with colorectal carcinoma, specifically KRAS and NRAS, has significant clinical implications. It is currently recommended that patients with colon cancer who are considered for antiepidermal growth factor receptor monoclonal antibodies, get RAS mutation testing since only those with wildtype-RAS genes benefit from such treatment. Despite decades of research, there is currently no effective and safe treatment that directly targets RAS-mutated neoplasms. Multiple therapeutic approaches directed against RAS mutations are currently experimental, including a promising immunotherapy study using T-cells in patients with metastatic colon cancer.

\section{INTRODUCTION}

In the USA, colorectal carcinoma is the third most common cancer in men and women and the second leading cause of cancer-related death. ${ }^{1}$ The current cancer statistics in the USA estimate that in 2018, 237470 men and women will be diagnosed with colorectal cancer and 50630 will die from it. ${ }^{1}$ Colorectal cancer develops because of a complex interaction between various genetic and environmental factors. ${ }^{23}$

Our understanding of the genetic alterations associated with the development and progression of colorectal carcinoma, in both hereditary and sporadic cases has tremendously improved over the last few decades. Colorectal cancer usually develops as a result of stepwise, multiple mutations involving oncogenes and tumour suppressor genes. Currently, the transcriptome data from a multitude of research helped categorise colorectal carcinoma into four molecular subtypes called consensus molecular subtypes (CMS). ${ }^{4}$ The first group, CMS1, is microsatellite instable and characterised by multiple mutations, hypermethylation and BRAF mutations. The consensus molecular subtypes 2, 3 and 4 (CMS2, CMS3 and CMS4) are chromosomally instable and differ based on their gene expression signal. Most KRAS-mutated colorectal cancers fall within the CMS3 category. ${ }^{4}$ The recurrent somatic genetic alterations in colon cancer lead to the development of neoplasia by affecting cells through different signalling routes. Genes of the RAS family are usually affected as part of the RTK-RAS signalling pathway. ${ }^{2}$ Mutations involving the RAS genes are seen in approximately $52 \%$ of colorectal carcinoma. $^{5}$

\section{RAS GENES AND PROTEINS IN THE NORMAL STATE}

The RAS family is formed of three genes, KRAS, NRAS and HRAS. ${ }^{6-8}$ Two of these genes were discovered more than four decades ago as retroviral oncogenes within two viruses: Kristen rat sarcoma virus (KRAS) and Harvey rat sarcoma virus (HRAS). ${ }^{9}$ NRAS (neuroblastoma RAS viral oncogene) was later discovered as the third member of the family. ${ }^{10}$

RAS genes code for four isoproteins: K-RAS4A, K-RAS4B, H-RAS and N-RAS, which share $82 \%-90 \%$ sequence identity (figure 1). ${ }^{6-8}$ These proteins act as a link in the intracellular signal transduction initiated by binding growth factors to cell membrane receptors. ${ }^{68}$ They help mediate signals related to cell survival, senescence and others. ${ }^{68}$ Despite their shared similarity, there is compelling evidence that RAS isoproteins have distinct functions in different tissues in both health state and cancer. $^{6-8}$ The differences in function are at least partially attributed to the differences in the C-terminal hypervariable region of these proteins.

RAS proteins are GTP-binding proteins with relatively weak GTPase functionality that allow these proteins to switch between two states: active (GTP-pound) and inactive (GDP-pound). ${ }^{6-8}$ This switching mechanism is supplemented by the actions of two classes of proteins: GTPase activating proteins, which helps to 'switch off' the signal by hydrolysing and releasing GTP and guanine nucleotide exchange factors, which 'switches on' the signal by favouring the formation of RAS-GDP complex. ${ }^{11}$

Although RAS proteins interact with several effectors, the two major signalling pathways through which RAS proteins exert their function are mitogen-activated protein kinase (MAPK) and phosphoinositide-3 kinase (PI3K). Discussing these pathways is beyond the scope of this review. Figure 2 shows a simplified summary of the RAS protein as a signal 

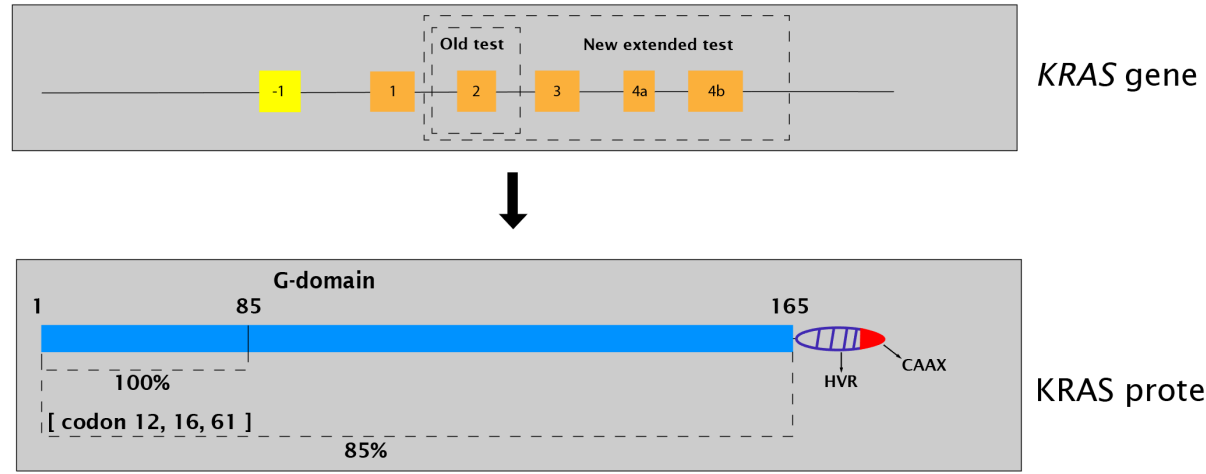

KRAS protein

Figure 1 The basic structure of the KRAS gene (upper diagram). The exons represented by the blue rectangles are the ones that code for proteins. Differential splicing to either include or exclude exon 4a results in the two KRAS isoforms: KRAS4A and KRAS4B. The recommended exons to be included in testing in the previous and current guidelines are highlighted. The lower diagram shows the basic KRAS protein structure with two domains; the G domain (GTP binding domain), which is similar between the four RAS isoproteins (per cent similarity shown, 100\% for first 85 amino acids and $\sim 85 \%$ for the whole $\mathrm{G}$ domain) and the hypervariable region with CAAX motif at the end, forming the membrane targeting part of the protein.

transducer and its interaction with the various intracellular effectors, focusing on the MAPK and PI3K pathways.

\section{RAS MUTATIONS AND COLORECTAL CARCINOMA PATHOGENESIS}

Mutations of RAS genes are among the first mutations to be discovered in malignant tumours. They still represent the most mutated genes in cancers, being found in approximately $25 \%$ of all human malignancies. ${ }^{7}$ RAS genes differ greatly in their mutational frequency among different malignancies with KRAS being the most commonly mutated. ${ }^{6}$ While KRAS represents approximately $100 \%$ of RAS mutations in pancreatic ductal carcinoma, NRAS accounts for $94 \%$ of RAS mutations in cutaneous melanoma. ${ }^{6}$ Despite the presumed functional differences between the three RAS isoforms in health and cancer pathogenesis, mutations affecting these genes usually involve the identical amino acid domains shared by the isoforms. ${ }^{6}$ Most of RAS mutations occur in only three codons: 12 , 13 (exon 2) and 61 (exon 3$)^{6}{ }^{6}$ The remaining few mutations usually involve codons 59 (exon 3) and codons 117, 146 (exon 4). ${ }^{12}$

KRAS has a contributory role in the development and progression of colorectal adenomas. This conclusion was supported by

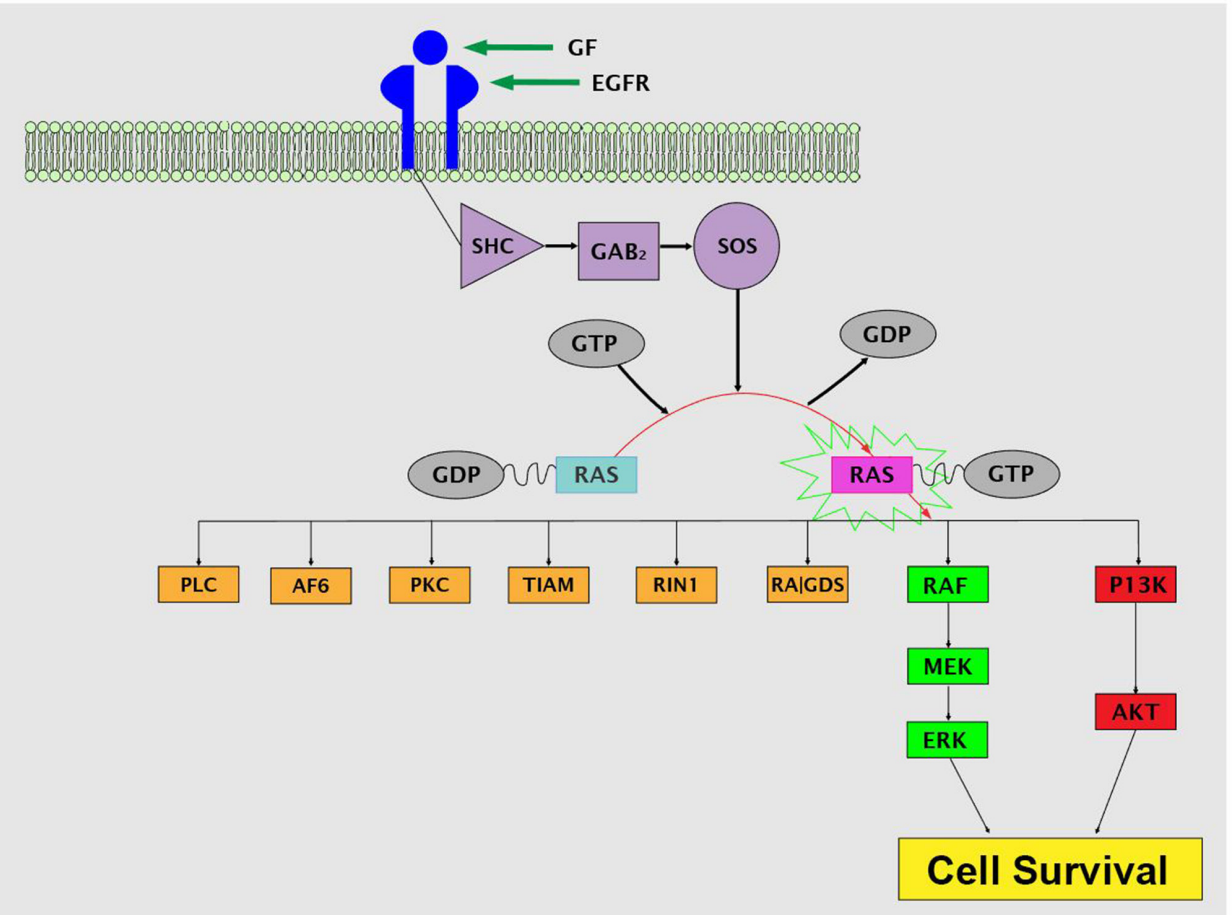

Figure 2 The role of RAS protein as a signal transducer connecting to multiple downstream effectors. The two downstream effector pathways commonly involved in colon cancer pathogenesis and known to mediate cell survival are further detailed. AF6, Afadin, AKT, protein kinase B; eGFR, epidermal growth factor receptor; ERK, extracellular signal-regulated kinase; Gab2: Grb2 associated binding protein 2; GDP, guanosine diphosphate, phospholipase C; GF, growth factor; GTP, guanosine-5'-triphosphate; MEK, mitogen-activated protein kinase; Pi3K, phosphatidylinositol $(3,4,5)$-trisphosphate; PKC, protein kinase C; RalGDS, Ral guanine nucleotide dissociation stimulator; RAF, rapidly accelerated fibrosarcoma; RIN1, RAS and RAB interactor-1; Shc, Src homolog and collagen; SOS, Son of Sevenless; TIAM, T-lymphoma invasion and metastasis inducing protein. 
demonstrating that KRAS mutations are more frequent in large adenomas compared with smaller ones. ${ }^{13}$

In the setting of colorectal carcinoma, both KRAS and NRAS mutations are frequently encountered in approximately $44.7 \%$ and $7.5 \%$ of cases, respectively. ${ }^{5}$ Most of KRAS mutations affect codon 12 and codon $13 .^{214}$ The essential role of KRAS mutations in the carcinogenesis of colorectal cancers has been illustrated by animal studies. In one study, although KRAS mutation alone was not sufficient to initiate colon cancer in mice, an APC-mutated mouse with secondary KRAS mutation developed a significantly larger number of adenocarcinomas with uniform high-grade dysplasia compared with those with wildtype-KRAS genes. ${ }^{15}$

In addition to the role of KRAS mutations in tumour progression, they seem to play an essential role in tumour maintenance. ${ }^{6-8}$ Loss of KRAS expression in animal models was associated with increased apoptosis in primary and metastatic colon cancers. ${ }^{16} 17$ KRAS-mutated colorectal cancers seem to have a specific clinicopathological profile. They tend to affect males and have classical adenocarcinoma morphology, are well or moderately differentiated and are microsatellite stable. ${ }^{14}$

\section{TESTING OF RAS MUTATIONS IN COLON CANCER: GUIDELINES AND NEW ADVANCES \\ Purpose of testing}

Testing for RAS mutations have a proven predictive value in patients with metastatic colon cancer. One treatment modality for patients with metastatic colon cancer is the addition of antiepidermal growth factor receptor (anti-EGFR) monoclonal antibodies, panitumumab and cetuximab, to the standard chemotherapy. These antibodies are known to improve outcome in patients with metastatic colorectal cancer and wildtype-RAS genes (lacking mutations in exons 2,3 and 4$){ }^{18}$

\section{Who should be tested}

The American Society for Clinical pathology, College of American Pathologists, Association for Molecular Pathology and the American Society of Clinical Oncology (ASCP/CAP/AMP/ASCO) issued joined guidelines for molecular testing in colorectal cancer in 2017. They recommend that all patients with colorectal cancer considered for anti-EGFR therapy receive extended testing for KRAS and NRAS codons 12, 13, 59, 61, 117 and 146 (table 1). ${ }^{19}$ The 2018 National Comprehensive Cancer Network (NCCN) guidelines for colon cancer recommends testing for RAS and BRAF in all patients with metastatic colorectal cancer. ${ }^{20}$

\section{Type of tissue needed for testing}

The NCCN and ASCP/CAP/AMP/ASCO guidelines recommend that either primary or metastatic tumour can be used for testing. ${ }^{20} 21$ They state in their consensus that both formalin-fixed,

\begin{tabular}{|c|c|c|c|}
\hline \multicolumn{3}{|c|}{ RAS testing (previous guidelines, 2009) } & \multirow{2}{*}{$\begin{array}{l}\text { Extended RAS testing (current } \\
\text { guidelines, 2017) } \\
\text { KRAS and NRAS }\end{array}$} \\
\hline KRAS & & & \\
\hline Exon & Codon & Exon & Codon \\
\hline \multirow[t]{6}{*}{2} & 12 & 2 & 12 \\
\hline & & & 13 \\
\hline & & 3 & 59 \\
\hline & 13 & & 61 \\
\hline & & 4 & 117 \\
\hline & & & 146 \\
\hline
\end{tabular}

paraffin-embedded tissue and cytology specimens after proper validation are appropriate for RAS mutation molecular testing. ${ }^{20} 21$

\section{Testing methodologies}

Various laboratory platforms exist for performing extended RAS testing including allele-specific PCR, PCR high-resolution melting assays, Sanger sequencing and next generation sequencing. These methodologies differ in sensitivity (ranging from $84.4 \%$ to $100 \%$ ) and specificity (ranging from $98 \%$ to $100 \%$ ). The joined guidelines of ASCP/CAP/AMP/ASCO do not favour any of these methodologies if the methodology can detect RAS mutation in specimens with low mutation frequency (5\%) (box 1). ${ }^{19} 22$

\section{Liquid biopsy as a modality for testing}

The detection of circulating tumour DNA (ctDNA) from a blood sample is convenient, in theory, and also provides a real-time detection of tumour burden, evolution and heterogeneity. It can be performed on almost every patient, even those with lesions difficult to biopsy. ${ }^{23-25}$ Recently, the utility of blood-based RAS testing (liquid biopsy) in patients with metastatic colorectal carcinoma showed great promise. ${ }^{25-29}$ In one study, the use of highly sensitive digital PCR (BEAMing) showed a $90.4 \%$ positive agreement, 93.5\% negative agreement and 91.8\% concordance compared with tissue-based RAS testing. ${ }^{30}$

Many studies have also shown that detecting KRAS mutation in liquid biopsy postoperatively in patients with colon cancer strongly predicts disease recurrence. ${ }^{25-27}$ Serial measurement and analysis of ctDNA in patients with colon cancer have also been shown to

\section{Box 1 Key points from the 2017 ASCO/AMP/CAP} guidelines regarding RAS testing in colorectal cancer

- RAS genes are the most commonly mutated genes in human malignancy, found in $25 \%$ of all cases.

- The four RAS isoproteins (KRASA4, KRASB4, NRAS and HRAS) share approximately $80 \%$ sequence identity, yet thought to have different roles in health and malignancy.

- RAS proteins act as a link in the signal transduction induced by growth factors to promote cell survival.

- KRAS and NRAS genes are mutated in $52 \%$ of patients with colon cancer, with most of the mutations affecting exon 2.

- KRAS mutations have a role in both the development and progression of colon cancer.

- According to the latest NCCN guidelines, testing for RAS mutations should be done for all patients with metastatic colon cancer disease.

- Testing is preferably performed on metastatic lesions using formalin-fixed, paraffin-embedded tissue. But cytology specimens can be used after proper validation.

- Testing should include mutations affecting exons 2, 3 and 4 in both KRAS and NRAS genes (extended RAS testing)

- Various testing platforms can be used if they are validated and meet clinical standards.

- Testing RAS mutations in liquid biopsy is showing great promise and might replace traditional tissue testing in the near future.

- Only patients with wildtype-RAS genes should be considered for anti-EGFR treatment.

- Even though decades of research have failed to produce an effective and safe RAS targeted therapy, promising clinical trials are on the way. 


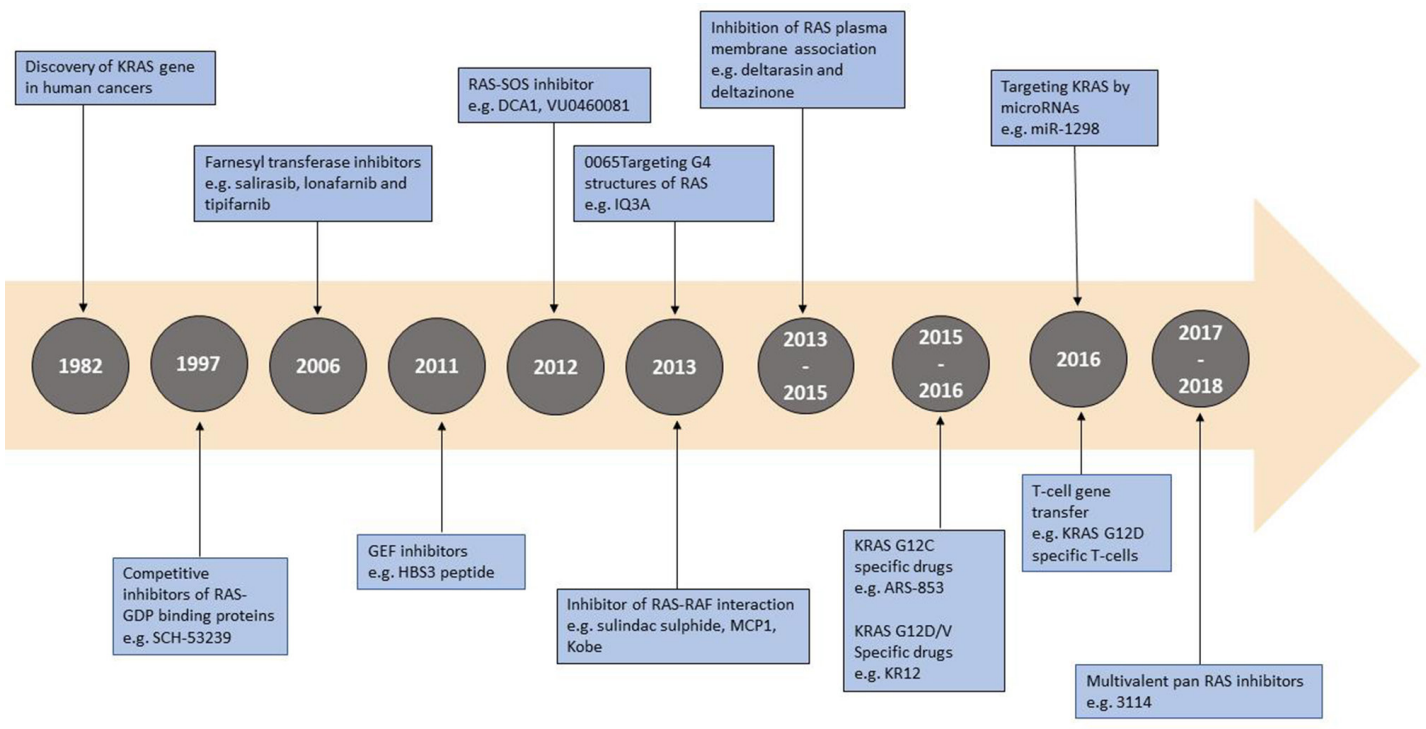

Figure 3 The timeline for the key direct RAS drugs since the discovery of RAS gene mutations in human malignancy and until now. This is not meant to be an exclusive list of the large number of experimental drugs that have been discovered, but a simplified timeline for the main drug and drug categories.

help both on the assessment of treatment response and the detection of the emergence of treatment resistance. ${ }^{27} 2830$

\section{KRAS MUTATIONS AS PROGNOSTIC BIOMARKER}

Several articles investigated the prognostic value of mutations affecting different codons and alternative mutations affecting the same codons of KRAS gene in the prognosis of patients with colon cancer. ${ }^{21}$ Patients with mutations involving codons 12 and 61 usually fare worse than those with other mutations, ${ }^{31-37}$ while mutations in codon 146 are associated with a better outcome. ${ }^{31}$ While the adverse prognostic effects of mutations involving exon 2 are supported by multiple studies, conflicting results regarding the differences in outcome between the common exon 2 mutated codons, 12 and 13, are seen in the literature. ${ }^{38}$ Unlike in pancreatic adenocarcinoma, in colorectal cancers, studies failed to show a significant outcome difference between G12D and G12V mutations affecting codon $2 .^{31}$

\section{RAS-TARGETED THERAPIES}

RAS proteins have always been thought of as potential therapeutic targets due to their critical role in the development of many malignancies including colorectal cancer. ${ }^{39-42}$ Despite that, more than three decades of research have failed to produce an effective and safe drug that can target RAS proteins. ${ }^{43}$ This failure is at least partially attributable to the biochemistry of RAS proteins. RAS proteins have a relatively smooth shape that hinder drug attachment, rendering direct targeted therapy difficult. ${ }^{43}$ Figure 3 shows some of the key categories of direct RAS drugs/experimental molecules and their timeline since RAS discovery until now.

Recent advances have shown that GTP is so tightly bound to RAS proteins that it is nearly impossible for small molecular inhibitors to displace GTP. ${ }^{44}$ Therefore, novel approaches to discover direct RAS inhibitors have been developed for codon 146 (figure 3). ${ }^{45-48}$ One of these approaches is to use drugs that target the mutated sites in the KRAS gene. These drugs hinder the formation of the GTP-bound activated KRAS proteins. Examples of these are experimental drugs that interfere with the reaction of the mutant KRAS G12D with SOS1 (one of the guanine nucleotide exchange protein) preventing the formation of KRAS-GTP. ${ }^{49}$ Others target the cysteine amino acid in KRAS G12C, blocking the activation of RAF and inducing apoptosis in tumours harbouring such mutations. $^{4748} 50$ Brito et al suggested another approach through targeting the guanine-rich G4 structure in the KRAS promotor region leading to decreased cell proliferation and increased apoptosis. ${ }^{51}$ A third interesting approach is the use of miRNA to silence and suppress KRAS-dependent cell growth both in vivo and in vitro. ${ }^{52}$ In 2017 , Welsch et al published their discovery of a small multivalent molecule capable of binding the active RAS-GTP protein and preventing the downstream interaction with effector proteins. As they explained in their article, this molecule showed toxic effect of target activity, necessitating further workup and development (box 2). ${ }^{53}$

A recent article in the New England Journal of Medicine showed a promising new approach to treating metastatic colorectal cancer using immunotherapy. ${ }^{54}$ The authors reported regression in a case of metastatic colon cancer after infusion with KRAS-mutant-specific T-cells, giving new hope for the use of immunotherapy as a potential treatment modality for metastatic colon cancer. ${ }^{54}$ Despite the tremendous efforts and extensive research over the last few decades, there is still no effective drug targeting mutated RAS genes whether directly or indirectly. ${ }^{55}$

\section{SUMMARY}

Mutations in KRAS and NRAS are seen in approximately 52\% of colorectal cancers, commonly involving codons 12, 13 and 61.

Box 2 Extended RAS testing in the current guidelines compared with previous recommended testing

- Testing should be done in all patients with metastatic colon cancer disease considered for anti-EGFR therapy.

- Testing is preferably performed on metastatic lesions using formalin-fixed, paraffin-embedded tissue or cytology specimens after proper validation.

- Testing should include mutations affecting exons 2, 3 and 4 in both KRAS and NRAS genes (extended RAS testing).

- Various testing platforms can be used if they are validated and meet clinical standards. 
RAS gene structures and functions have been thoroughly studied and described since their discovery decades ago. Their role in the pathogenesis of malignancy, including colon cancer cases, has been supported by many studies including animal models. The extended testing for KRAS and NRAS mutations is now the standard of care in patients with stage IV colon cancer considered for antiEGFR monoclonal antibodies. Despite decades of futile attempts to synthesise a safe and effective RAS targeted therapy, the last few years showed a renewed interest and significant progress on new and novel approaches to target KRAS mutated tumours.

Correction notice This paper has been corrected since it was published Online First. Figure 2 and 3 legends were accidentally switched and this has now been rectified.

Handling editor Des Richardson.

Contributors OS drafted the article. LC formulated and designed the topic of this review. All the authors critically read, edited and approved the final manuscript.

Funding The authors have not declared a specific grant for this research from any funding agency in the public, commercial or not-for-profit sectors.

Competing interests None declared.

Patient consent Not required.

Provenance and peer review Not commissioned; externally peer reviewed.

\section{REFERENCES}

1 Siegel RL, Miller KD, Jemal A. Cancer statistics. CA J Clin Phys 2018;2018:7-30.

2 Fearon ER. Molecular genetics of colorectal cancer. Annu Rev Pathol 2011;6:479-507.

3 Kuipers EJ, Grady WM, Lieberman D, et al. Colorectal cancer. Nat Rev Dis Primers 2015;1:15065.

4 Dienstmann R, Vermeulen L, Guinney J, et al. Consensus molecular subtypes and the evolution of precision medicine in colorectal cancer. Nat Rev Cancer 2017;17:79-92.

5 Cox AD, Fesik SW, Kimmelman AC, et al. Drugging the undruggable RAS: Mission possible? Nat Rev Drug Discov 2014;13:828-51.

6 Prior IA, Lewis PD, Mattos C. A comprehensive survey of ras mutations in cancer. Cancer Res 2012;72:2457-67.

7 Papke B, Der CJ. Drugging RAS: know the enemy. Science 2017;355:1158-63.

8 Jinesh GG, Sambandam V, Vijayaraghavan S, et al. Molecular genetics and cellular events of K-Ras-driven tumorigenesis. Oncogene 2018;37:839-46.

9 Rauen KA. The RASopathies. Annu Rev Genomics Hum Genet 2013;14:355-69

10 Malumbres M, Barbacid M. RAS oncogenes: the first 30 years. Nat Rev Cancer 2003;3:459-65.

11 Pylayeva-Gupta Y, Grabocka E, Bar-Sagi D. RAS oncogenes: weaving a tumorigenic web. Nat Rev Cancer 2011;11:761-74.

12 Al-Shamsi HO, Alhazzani W, Wolff RA. Extended RAS testing in metastatic colorectal cancer-Refining the predictive molecular biomarkers. J Gastrointest Oncol 2015;6:314.

13 Vogelstein B, Fearon ER, Hamilton SR, et al. Genetic alterations during colorectal-tumor development. N Eng/ J Med 1988;319:525-32.

14 Rimbert J, Tachon G, Junca A, et al. Association between clinicopathological characteristics and RAS mutation in colorectal cancer. Mod Pathol 2018:31:517-26.

15 Haigis KM, Kendall KR, Wang Y, et al. Differential effects of oncogenic K-Ras and $\mathrm{N}$-Ras on proliferation, differentiation and tumor progression in the colon. Nat Genet 2008;40:600-8.

16 Stephen AG, Esposito D, Bagni RK, et al. Dragging ras back in the ring. Cancer Cell 2014;25:272-81.

17 Simanshu DK, Nissley DV, McCormick F. RAS proteins and their regulators in human disease. Cell 2017;170:17-33.

18 Douillard JY, Oliner KS, Siena S, et al. Panitumumab-FOLFOX4 treatment and RAS mutations in colorectal cancer. N Engl J Med 2013;369:1023-34.

19 Sepulveda AR, Hamilton SR, Allegra CJ, et al. Molecular biomarkers for the evaluation of colorectal cancer: guideline from the american society for clinical pathology, college of american pathologists, association for molecular pathology, and the american society of clinical oncology. J Clin Oncol 2017;35:1453-86.

20 NCCN clinical practice guidelines in oncology (NCCN guidelines): colon cancer Version 2. Vol. 2018, 2018.

21 Allegra CJ, Rumble RB, Hamilton SR, et al. Extended RAS gene mutation testing in metastatic colorectal carcinoma to predict response to anti-epidermal growth factor receptor monoclonal antibody therapy: American society of clinical oncology provisional clinical opinion update 2015. J Clin Oncol 2016:34:179-85.

22 Van Krieken JH, Rouleau E, Ligtenberg MJ, et al. RAS testing in metastatic colorectal cancer: advances in Europe. Virchows Arch 2016;468:383-96.

23 Wan JCM, Massie C, Garcia-Corbacho J, et al. Liquid biopsies come of age: towards implementation of circulating tumour DNA. Nat Rev Cancer 2017:17:223-38.
24 Merker JD, Oxnard GR, Compton C, et al. Circulating tumor DNA analysis in patients with cancer: American society of clinical oncology and college of American pathologists joint review. J Clin Oncol 2018;36:1631-41.

25 Strickler JH, Loree JM, Ahronian LG, et al. Genomic landscape of Cell-Free DNA in patients with colorectal cancer. Cancer Discov 2018;8:164-73.

26 Cohen JD, Li L, Wang Y, et al. Detection and localization of surgically resectable cancers with a multi-analyte blood test. Science 2018;359:926-30.

27 Tie J, Wang Y, Tomasetti C, et al. Circulating tumor DNA analysis detects minimal residual disease and predicts recurrence in patients with stage II colon cancer. Sci Trans/ Med 2016:8:ra92

28 Garlan F, Laurent-Puig P, Sefrioui D, et al. Early evaluation of circulating tumor DNA as marker of therapeutic efficacy in metastatic colorectal cancer patients (PLACOL Study). Clin Cancer Res 2017:23:5416-25.

29 Thierry AR, El Messaoudi S, Mollevi C, et al. Clinical utility of circulating DNA analysis for rapid detection of actionable mutations to select metastatic colorectal patients for antiEGFR treatment. Ann Oncol 2017;28:2149-59.

30 Schmiegel W, Scott RJ, Dooley S, et al. Blood-based detection of RAS mutations to guide anti-EGFR therapy in colorectal cancer patients: concordance of results from circulating tumor DNA and tissue-based RAS testing. Mol Oncol 2017;11:208-19.

31 Haigis KM. Kras alleles: the devil is in the detail. Trends Cancer 2017;3:686-97.

32 Taieb J, Le Malicot K, Shi Q, et al. Prognostic value of BRAF and KRAS Mutations in MSI and MSS stage III colon cancer. J Natl Cancer Inst 2017;109:djw272.

33 Jones RP, Sutton PA, Evans JP, et al. Specific mutations in KRAS codon 12 are associated with worse overall survival in patients with advanced and recurrent colorectal cancer. $\mathrm{Br} J$ Cancer 2017;116:923-9.

34 Morris VK, Lucas FA, Overman MJ, et al. Clinicopathologic characteristics and gene expression analyses of non-KRAS 12/13, RAS-mutated metastatic colorectal cancer. Ann Oncol 2014:25:2008-14.

35 Cserepes M, Ostoros G, Lohinai Z, et al. Subtype-specific KRAS mutations in advanced lung adenocarcinoma: a retrospective study of patients treated with platinum-based chemotherapy. Eur J Cancer 2014;50:1819-28.

36 Mao C, Huang YF, Yang ZY, et al. KRAS p.G13D mutation and codon 12 mutations are not created equal in predicting clinical outcomes of cetuximab in metastatic colorectal cancer: a systematic review and meta-analysis. Cancer 2013;119:714-21.

37 Tejpar S, Celik I, Schlichting M, et al. Association of KRAS G13D tumor mutation with outcome in patients with metastatic colorectal cancer treated with first-line chemotherapy with or without cetuximab. J Clin Oncol 2012:30:3570-7.

38 Auclin E, Zaanan A, Vernerey D, et al. Subgroups and prognostication in stage III colon cancer: future perspectives for adjuvant therapy. Ann Oncol 2017:28:mdx030-68.

39 Liu Y, Sethi NS, Hinoue T, et al. Comparative molecular analysis of gastrointestinal adenocarcinomas. Cancer Cell 2018;33:721-35.

40 Cheng L, Lopez-Beltran A, Massari F, et al. Molecular testing for BRAF mutations to inform melanoma treatment decisions: a move toward precision medicine. Mod Pathol 2018:31:24-38.

41 Roerink SF, Sasaki N, Lee-Six H, et al. Intra-tumour diversification in colorectal cancer at the single-cell level. Nature 2018;556:457-62.

42 Misale S, Di Nicolantonio F, Sartore-Bianchi A, et al. Resistance to anti-EGFR therapy in colorectal cancer: from heterogeneity to convergent evolution. Cancer Discov 2014:4:1269-80

43 Ledford H. Cancer: The Ras renaissance. Nature 2015:520:278-80.

44 Müller MP, Jeganathan S, Heidrich A, et al. Nucleotide based covalent inhibitors of KRas can only be efficient in vivo if they bind reversibly with GTP-like affinity. Sci Rep 2017:7:3687.

45 Mai TT, Lito P. A treatment strategy for KRAS-driven tumors. Nat Med 2018;24:902-4.

46 Dang CV, Reddy EP, Shokat KM, et al. Drugging the 'undruggable' cancer targets. Nat Rev Cancer 2017;17:502-8.

47 Bahrami A, Hassanian SM, ShahidSales S, et al. Targeting RAS signaling pathway as a potential therapeutic target in the treatment of colorectal cancer. J Cell Physiol 2018:233:2058-66.

48 Román M, Baraibar I, López I, et al. KRAS oncogene in non-small cell lung cancer: clinical perspectives on the treatment of an old target. Mol Cancer 2018:17:33.

49 Maurer T, Garrenton LS, Oh A, et al. Small-molecule ligands bind to a distinct pocket in Ras and inhibit SOS-mediated nucleotide exchange activity. Proc Natl Acad Sci U S A 2012:109:5299-304.

50 Janes MR, Zhang J, Li LS, et al. Targeting KRAS mutant cancers with a covalent G12C specific inhibitor. Cell 2018:172:578-89.

51 Brito H, Martins AC, Lavrado J. Targeting KRAS oncogene in colon cancer cells with 7-carboxylate indolo[3,2-b]quinoline tri-alkylamine deriv Derivatives. PLoS One 2015;10:e0126891.

52 Zhou Y, Dang J, Chang KY, et al. miR-1298 inhibits mutant KRAS-driven tumor growth by repressing FAK and LAMB3. Cancer Res 2016;76:5777-87.

53 Welsch ME, Kaplan A, Chambers JM, et al. Multivalent small-molecule Pan-RAS inhibitors. Cell 2017:168:878-89.

54 Tran E, Robbins PF, Lu YC, et al. T-cell transfer therapy targeting mutant KRAS in cancer. N Engl J Med 2016:375:2255-62.

55 Fisher KW, Lopez-Beltran A, Montironi R, et al. Precision medicine in colorectal cancer: evolving genomic landscape and emerging consensus. Future Oncol 2015:11:2711-9. 